\title{
Analysis of On-trial Quality Assurance for the SPARC Clinical Trial using Novel Peer-review Methodology
}

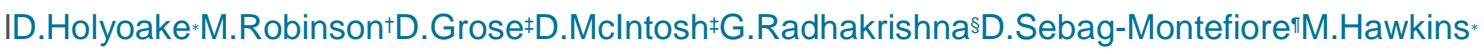

Aims/objectives: Pre-trial radiotherapy quality assurance (RTQA) for the SPARC trial of preoperative pancreas SBRT highlighted overgenerous target contouring [1]. We aimed to assess for improvement following a training workshop and appraise the effectiveness of a novel web-conferencing format for on-trial RTQA.

Content of presentation: The results of on-trial RTQA, performed utilising webconferencing, were analysed, for all patients, patients recruited to the study to date (11). Frequency of requirement for changes was assessed and results compared to those for pre-trial RTQA.

Relevance/impact: Prompt review of contours and plans using web-conferencing enables rapid peer feedback with approval or correction to reduce the risk of delays in RTQA.

Outcomes: For seven cases contours were approved immediately. In four cases the target structures were not acceptable, in three this was due to the GTV contour including non-tumour tissue, and in one case the structure was corrected live and approved. In two cases the boost target structure was inaccurate. These results compare favourably with those from pre-trial RTQA in which only two of eight submitted GTV contours were acceptable, although in all but two cases the boost volumes were acceptable. For eight cases (72\%) the treatment plan was approved without concern. For two cases advice was given for improvement of planning for subsequent cases. For one case changes were requested for the current clinical plan, relating to the conformity of SBRT dose hotspot to PTV.

Discussion: Target contour definition for trial patients was improved compared to pre-trial, but overgenerous contouring remains an issue. 